\title{
Single-Tube Genotyping from a Human Hair Root by Direct PCR
}

\author{
Mariko Hayashida, Kyoko Iwao-KoIzumi, Shigenori Murata, and Kenji Kinoshita ${ }^{\dagger}$ \\ School of Pharmaceutical Sciences, Mukogawa Women's University, Koshien, Nishinomiya 663-8179, Japan
}

\begin{abstract}
We have developed a simple, labor-saving, inexpensive and rapid SNP genotyping method that directly uses a human hair root as the template. This single-tube genotyping method was used to successfully and reliably genotype the $A D H 1 B$ and $A L D H 2$ polymorphisms using a hair root (without DNA isolation) and the polymerase chain reaction (PCR) enzyme kit KOD FX. Since the DNA extraction step was eliminated, the possibility of sample contamination was considerably decreased. The single-tube SNP genotyping was performed by coupling the PCR enzyme kit with allele-specific primer (ASP)-PCR. In the post-genome era, a simple and inexpensive method for diagnostic analysis is in high demand, and this PCR method with a hair root as a template will be very useful for genetic diagnoses in biological and medical laboratories.
\end{abstract}

(Received October 23, 2009; Accepted October 27, 2009; Published December 10, 2009)

\section{Introduction}

SNP genotyping methods are becoming increasingly important for disease diagnosis. ${ }^{1}$ Various assays have been developed to analyze SNP genotypes, including direct DNA sequencing after polymerase chain reaction (PCR) amplification, ${ }^{2} \mathrm{PCR}-$ restriction fragment length polymorphisms (PCR-RFLP), ${ }^{3}$ TaqMan PCR, ${ }^{4}$ and allele-specific primer-PCR (ASP-PCR). ${ }^{5}$ SNP genotyping can generally be divided into two steps: sample preparation, e.g., purification of DNA from various biological specimens such as blood, and allele detection. The most basic method to isolate DNA from biological materials requires multiple steps, including Pro-K/detergent treatment such as with SDS, phenol/chloroform extraction, cold ethanol precipitation, and sample reconstitution in buffer. Generally, this process is labor-intensive, time-consuming, costly, and it enhances the risk of cross-contamination.

Recently, in order to save the time and expense required to purify the template DNA, several simplified protocols and kits have been developed that perform PCR amplification without DNA extraction from whole blood or dried blood spotted on filter paper.6-11 Human hair is also known to contain genomic DNA and represents a potentially noninvasive DNA source. Hair DNA, especially when obtained from the terminal hair roots, is very limited in quantity. To overcome this challenge, we developed an improved genotyping system that uses a direct PCR analysis of terminal hair root samples. The PCR amplification enzyme kit KOD FX, which is based on a novel KOD DNA polymerase from the Thermococcus kodakaraensis KOD1, effectively neutralizes inhibitors present in biological specimens. In addition, this system is conducive for reproducible and high-throughput direct PCR amplification of samples without DNA extraction from whole blood dried on paper. KOD FX results in much greater PCR success because it has a higher efficiency and elongation capability than other Taq-based PCR enzymes. Using this system, SNP genotypes could be

† To whom correspondence should be addressed.

E-mail: kenji_k@mukogawa-u.ac.jp determined from PCR products that used genomic DNA from blood dried on paper and stored for over 6 months at room temperature.

\section{Experimental}

\section{Subjects}

Twenty five healthy, non-pregnant female students as volunteers at Mukogawa Women's University aged 18-20 $(19 \pm 0.5)$ years who were able to attend the detailed instruction, and participated in the study. The Medical Ethics Committee of Mukogawa Women's University approved the study protocol and each woman provided written, informed consent to participate in all study.

\section{Study design}

In this study we developed a single-tube genotyping method that analyzes the hair root by direct PCR. Post-PCR steps and expensive instruments are not required. The type of SNP could be determined by the length of allele-specific amplicons in a single-tube PCR reaction with a hair root as a template. As shown in Fig. 1, the procedure is very simple, and all of the necessary PCR reagents as well as the hair root sample were added to the tube prior to the PCR. Therefore, the aim of the present study is to demonstrate an inexpensive and highthroughput genotyping method for detecting polymorphisms of alcohol dehydrogenase subunit $\beta(A D H 1 B)$ - and aldehyde dehydrogenase $2(A L D H 2)$-related SNPs by duplex ASP-PCR using a hair root as the starting material. If a hair root is directly used for ASP-PCR, the possibility of sample contamination could be considerably decreased. To our knowledge, this is the first report of a method that simultaneously detects multiple genetic polymorphisms using duplex ASP-PCR without any prior DNA purification of the hair root.

\section{Genotyping}

SNP genotyping of the $A D H 1 B$ (Arg47His) and $A L D H 2$ (Glu487Lys) genes was performed with standard thin-walled PCR tubes using an allele-specific primer mixture containing 

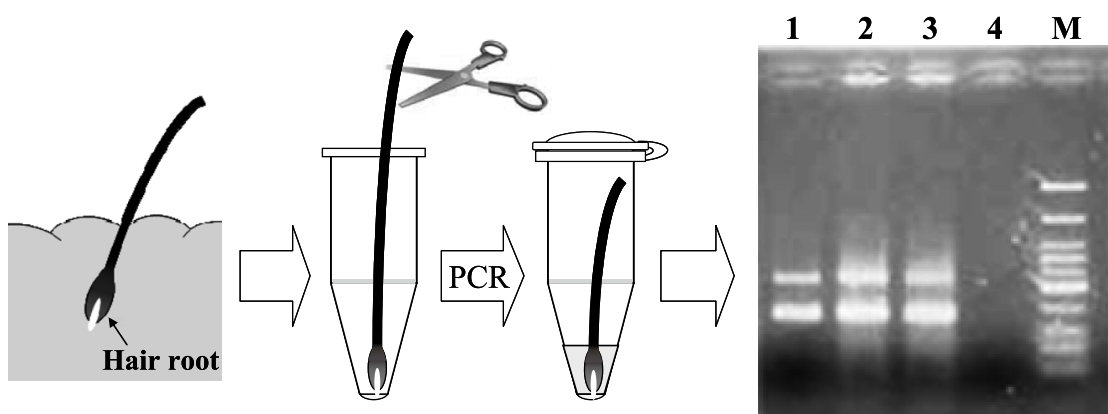

Fig. 1 Outline of the single-tube genotyping method using a human hair root and direct PCR. Gel image of $A D H 1 B$ and $A L D H 2$ SNP genotyping using duplex ASP-PCR for allele [G]. The electrophoresis represents reactions using human blood dried on filter paper or hair roots from the same person as templates. Lane M, 100 bp ladder. Lane: 1, blood dried on filter paper $(2 \mathrm{~mm} \phi) ; 2$, hair root sample 1; 3, hair root sample 2; 4, negative control (DW).
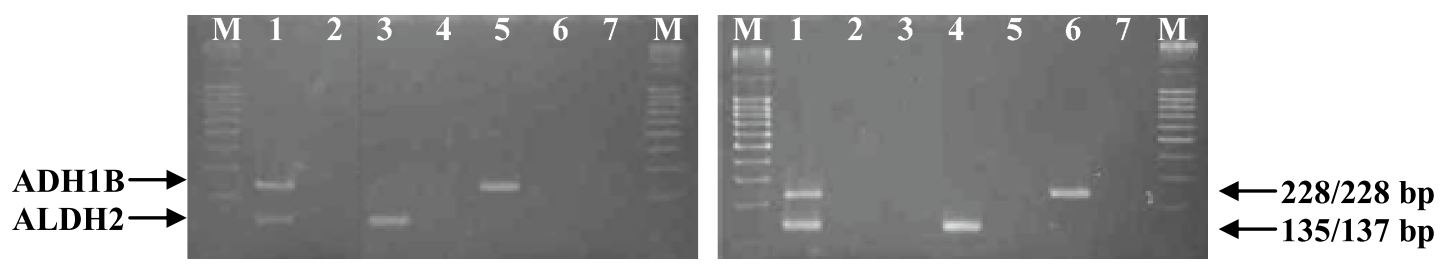

Fig. 2 Detection of $A D H 1 B$ and $A L D H 2$ SNP genotyping. Schematic representation of the PCR products generated using duplex ASP-PCR for allele [G] (left; ADH1B-F, ADH1B-R-G, ALDH2-F and ADH1B-R-G) and allele [A] (right; ADH1B-F, ADH1B-R-A, ALDH2-F and ADH1B-R-A). Lane M, 100 bp ladder. Lane 1, Human hair root sample, which has been genotyped as heterozygous for both $A D H 1 B$ and $A L D H 2$ using the direct DNA sequencing method. Lanes 2 and 7, negative control (DW). Lane: 3, positive control plasmid for $A L D H 2$ [G]; 4, positive control plasmid for $A L D H 2$ [A]; 5, positive control plasmid for $A D H 1 B$ [G]; 6, positive control plasmid for $A D H 1 B$ [A].

forward and reverse primers for each assay in the SNP typing kit on a Gene Amp PCR System 9700 (Applied Biosystems). SNP genotyping kits and positive controls, $A D H 1 B$ (STK-161, STK-161P, TOYOBO, Osaka, Japan) and $A L D H 2$ (STK-162, STK-162P, TOYOBO) for ASP-PCR, were obtained from TOYOBO. The amplification conditions were as follows: an initial denaturing step of $95^{\circ} \mathrm{C}$ for $5 \mathrm{~min}$, followed by 35 cycles of $98^{\circ} \mathrm{C}$ for $10 \mathrm{~s}, 64^{\circ} \mathrm{C}$ for $30 \mathrm{~s}$, and $74^{\circ} \mathrm{C}$ for $30 \mathrm{~s}$ and a final elongation step of $72^{\circ} \mathrm{C}$ for $2 \mathrm{~min}$. $A D H 1 B$ and $A L D H 2$ mutations were determined by ASP-PCR. PCR primers were designed for SNPs of the $A D H 1 B$ and $A L D H 2$ genes. The regions of the genes containing the single nucleotide polymorphisms were amplified by duplex PCR with four allele-specific primers for $A D H 1 B$ (ADH1B-F; 5'-GGTAGAGAAGGGCTTTAGACTGA-3', G-specific primer (ADH1BR-G); 5'-AACCACGTGGTCATCTGTAcG-3'; A-specific primer (ADH1B-R-A); 5'-AACCACGTGGTCATCTGTTtG-3', amplicon; W/M, 228/228 bp) and ALDH2 (ALDH2-F; 5'-TCAAATTACAGGGTCAACTGCT-3', G-specific primer (ALDH2-R-G); 5' $\quad$-CACACTCACAGTTTTCACTGcA-3'; A-specific primer(ALDH2-R-A); 5'-CCCACACTCACAGTTTTCACTAtA-3', amplicon; W/M, 135/137 bp). W, M, F, and R represent wild type, mutant, forward primer, and reverse primer, respectively. The penultimate nucleotide that distinguishes the wild type and mutant sequences is represented in lowercase letters. The 1-base pair mismatches at the antepenultimate nucleotide are underlined. Duplex ASP-PCRs were performed in a $25-\mu \mathrm{L}$ volume with the hair root. The reaction mixture contained $8.5 \mu \mathrm{L}$ of distilled water, $12.5 \mu \mathrm{L}$ of $2 \times$ PCR buffer for KOD FX, $2.5 \mu \mathrm{L}$ of $2 \mathrm{mM}$ dNTPs, $0.5 \mu \mathrm{L}$ each for the primer mixtures for allele [G] (ADH1B-F, ADH1B-R-G, ALDH2-F and ADH1B-R-G) or allele [A] (ADH1B-F, ADH1BR-A, ALDH2-F and ADH1B-R-A) in $A D H 1 B$ and $A L D H 2$, and $0.5 \mathrm{U}$ of KOD FX DNA polymerase $(1 \mathrm{U} / \mu \mathrm{L}$, KFX-101, TOYOBO). The final PCR products were analyzed by $3 \%$ agarose gel electrophoresis with $0.5 \mathrm{mg} / \mathrm{L}$ ethidium bromide in the electrophoresis chamber. Photographic images were captured using ChemiDoc XRS (BIO-RAD, Tokyo, Japan).

\section{Results and Discussion}

The results of our study, shown in Fig. 2, demonstrate that direct PCR amplification with the commercially available KOD FX buffer system and the hair root as a template could be successfully performed in a single tube. Figure 3 shows combined effects of $A D H 1 B$ and $A L D H 2$ genotypes among the female students as volunteers, and data with respect to the genotype distribution is shown in Table 1. In addition, this assay was so sensitive that two hair roots were enough to obtain a successful typing. This PCR assay eliminated the DNA extraction process, which is too laborious, costly, and timeconsuming for practical use. In addition, this PCR method addresses personal privacy protection concerns, as excess human genomic DNA does not remain after the SNP genotyping. In terms of transportation and storage, it is very convenient to use hair as a DNA source because no refrigeration is required. For this reason, hair samples can be collected in remote areas and 
$\begin{array}{llllllllllllllllllllllllll}1 & 2 & 3 & 4 & 5 & 6 & 7 & 8 & 9 & 10 & 11 & 12 & 13 & 14 & 15 & 16 & 17 & 18 & 19 & 20 & 21 & 22 & 23 & 24 & 25 & M\end{array}$

(A)

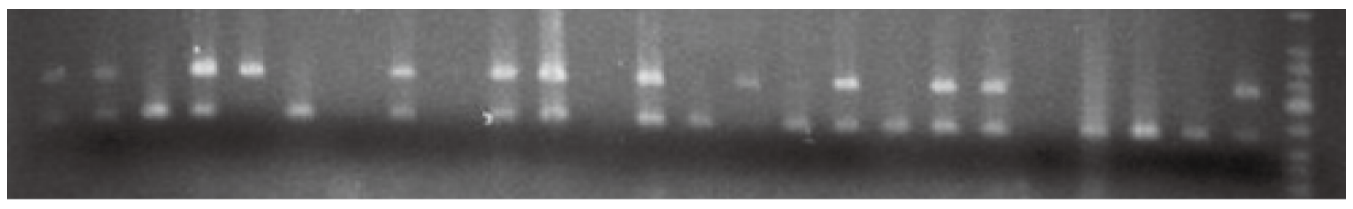

(B)

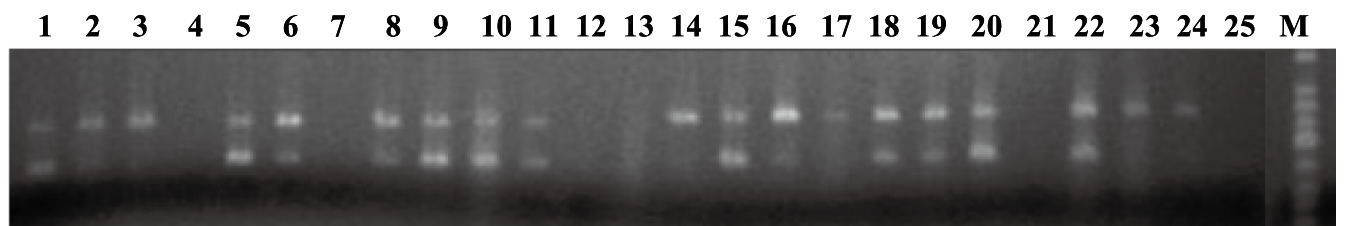

Fig. 3 Genotyping of $A D H 1 B$ and $A L D H 2$ SNPs among all volunteers. Schematic representation of the PCR products generated using duplex ASP-PCR. (A) Allele [G] for $A D H 1 B$ and $A L D H 2$. (B) Allele [A] for $A D H 1 B$ and $A L D H 2$. Lane M, 100 bp ladder. Lanes 1 to 25: Number of 25 volunteers. Lanes 7, 12, and 21: ND.

Table 1 Genotype distribution of $A D H 1 B$ and $A L D H 2$ in all volunteers

\begin{tabular}{cccc}
\hline$A D H 1 B$ & $A L D H 2$ & $n$ & Volunteer \\
\hline G/G & G/G & 3 & $4,13,25$ \\
G/G & G/A & 0 & \\
G/G & A/A & 0 & 17 \\
G/A & G/G & 1 & $1,2,8,10,11,19,20$ \\
G/A & G/A & 7 & 5,15 \\
G/A & A/A & 2 & $3,14,23,24$ \\
A/A & G/G & 4 & $6,16,18,22$ \\
A/A & G/A & 4 & 9 \\
A/A & A/A & 1 & \\
\hline
\end{tabular}

subsequently analyzed.

In conclusion, we have developed a highly accurate SNP genotyping method that uses only conventional equipment: a PCR thermocycler, electrophoresis instrument, and an illuminometer. This assay can detect both mutant alleles with point mutations and those with large structural alterations in less than $3 \mathrm{~h}$. Consequently, ASP-PCR using a commercially available DNA polymerase kit, KOD FX, is a true single-tube SNP typing method because all of the reagents and the unprocessed hair root samples are added simultaneously and no additional steps are required before the PCR.

\section{Acknowledgements}

We thank the anonymous volunteers who participated in the hair sample collection, and Ms. Takako Yamaguchi and Ms. Tomoko Ohta for her excellent technical support.

\section{References}

1. The International SNP Map Working Group, Nature, 2001, 409, 928.

2. T. H. Kim, T. J. Kim, H. S. Lee, W. S. Uhm, E. S. Shin, Y. I. Na, and J. B. Jun, J. Rheumatol., 2008, 35, 1849.

3. K. Obayashi, K. Nakamura, J. Kawana, H. Ogata, K. Hanada, M. Kurabayashi, A. Hasegawa, K. Yamamoto, and R. Horiuchi, Clin. Pharmacol. Ther., 2006, 80, 169.

4. P. Borgiani, C. Ciccacci, V. Forte, E. Sirianni, L. Novelli, P. Bramanti, and G. Novelli, Pharmacogenomics, 2009, 10, 261.

5. A. Ishiguro, T. Kubota, Y. Soya, H. Sasaki, O. Yagyu, Y. Takarada, and T. Iga, Anal. Biochem., 2005, 337, 256.

6. S. Raskin, J. A. 3rd Phillips, G. Kaplan, M. McClure, and C. Vnencak-Jones, PCR Methods Appl., 1992, 2, 154.

7. J. Burckhardt, PCR Methods Appl., 1994, 3, 239.

8. N. Nishimura, T. Nakayama, H. Tonoike, K. Kojima, Y. Shirasaki, K. Kondoh, and T. Yamada, Clin. Lab., 2002, 48, 377.

9. N. Nishimura, T. Nakayama, H. Tonoike, K. Kojima, and S. Kato, Ann. Clin. Biochem., 2000, 37, 674.

10. Y. G. Yang, J. Y. Kim, Y. H. Song, and D. S. Kim, Clin. Chim. Acta, 2007, 380, 112.

11. Y. Bu, H. Huang, and G. Zhou, Anal. Biochem., 2008, 375, 370. 